\title{
The slow-mode nature of compressible wave power in solar wind turbulence
}

\author{
G. G. Howes,${ }^{1}$ S. D. Bale, ${ }^{2,3}$ K. G. Klein, ${ }^{1}$ C. H. K. Chen, ${ }^{2}$ C. S. Salem, ${ }^{2}$ and J. M. TenBarge ${ }^{1}$ \\ ${ }^{1}$ Department of Physics and Astronomy, University of Iowa, Iowa City, Iowa 52242, USA. \\ ${ }^{2}$ Space Sciences Laboratory, University of California, Berkeley, California 94720-7450, USA. \\ ${ }^{3}$ Department of Physics, University of California, Berkeley, California, 94720-7300, USA.
}

(Dated: June 7, 2018)

\begin{abstract}
We use a large, statistical set of measurements from the Wind spacecraft at 1 AU, and supporting synthetic spacecraft data based on kinetic plasma theory, to show that the compressible component of inertial range solar wind turbulence is primarily in the kinetic slow mode. The zero-lag cross correlation $C\left(\delta n, \delta B_{\|}\right)$between proton density fluctuations $\delta n$ and the field-aligned (compressible) component of the magnetic field $\delta B_{\|}$is negative and close to -1 . The typical dependence of $C\left(\delta n, \delta B_{\|}\right)$on the ion plasma beta $\beta_{i}$ is consistent with a spectrum of compressible wave energy that is almost entirely in the kinetic slow mode. This has important implications for both the nature of the density fluctuation spectrum and for the cascade of kinetic turbulence to short wavelengths, favoring evolution to the kinetic Alfvén wave mode rather than the (fast) whistler mode.

PACS numbers: 96.50.Ci, 94.05.Lk, 52.35.Ra
\end{abstract}

Introduction.- The inertial range of solar wind turbulence is comprised of a mixture of incompressible and compressible motions, with at least $90 \%$ of the energy due to the incompressible component [1]. If these fluctuations are interpreted as some mixture of the three MHD linear wave modes, then Alfvén waves are the dominant incompressible component, while slow and fast MHD waves make up the compressible component. These modes are distinguished by the correlation between the density and parallel magnetic field fluctuations: fast waves are positively correlated, slow waves are negatively correlated, and the density and parallel magnetic field fluctuations are both zero for Alfvén waves. As the wave amplitude is increased to nonlinear levels, even to the limit that they form discontinuities or shocks, these qualitative properties persist, corresponding to tangential and rotational discontinuities or fast and slow shocks [2]. The MHD limit of strong collisionality, however, is not valid in the solar wind; therefore, collisionless kinetic theory is necessary to determine the properties of the wave modes. Each of the kinetic versions of the MHD linear wave modes, determined using the Vlasov-Maxwell linear dispersion relation, retain the qualitative correlations between the density and parallel magnetic field fluctuation described above [3]. In addition, these kinetic counterparts to the compressible modes may suffer damping from collisionless mechanisms [4].

Compressible fluctuations at inertial range scales $\lambda$ in the solar wind $\left(10^{-4} \mathrm{~Hz} \lesssim f_{s c} \sim v_{s w} / \lambda \lesssim 1 \mathrm{~Hz} ; v_{s w}\right.$ is the solar wind speed, $f_{s c}$ is the Doppler-shifted frequency in the spacecraft frame) have been studied extensively, often interpreted as a mix of magnetoacoustic (fast MHD) waves and pressure-balanced structures (PBSs) [1, 5]. A PBS was first observed as an anti-correlation of thermal pressure and magnetic pressure at timescales of $1 \mathrm{~h}[6]$, and subsequent investigations found a similar anti-correlation between the density and magnetic field magnitude [7, 8]. Theoretical studies of compressible MHD fluctuations in the low-Mach number, high- $\beta$ limit interpreted these anti-correlated density-magnetic field strength observations as nonpropagating "pseudosound" density fluctuations [9]. A more comprehensive investigation confirmed the general density-magnetic field anticorrelation, but also identified a few positively correlated intervals consistent with the magnetosonic (fast MHD) wave [10]. Analysis of Ulysses observations found evidence for PBSs at inertial range scales in the high latitude solar wind 11 13. Studies of the electron density up to $f_{s c}=2.5 \mathrm{~Hz}$ also found pressure balanced structures, but interpreted these as ion acoustic (slow MHD) waves, and recognized that PBSs are simply the ion acoustic (slow MHD) wave in the perpendicular wavevector limit [14]. Recently, measurements of the anti-correlation between electron density and magnetic field strength indicated the existence of PBSs over timescales ranging from $10^{3} \mathrm{~s}$ down to $10 \mathrm{~s}[15]$.

This Letter demonstrates that the compressible fluctuations in the inertial range are almost entirely kinetic slow wave fluctuations, suggesting that little turbulent energy is transferred from large scales to whistler fluctuations below the ion gyroscale. First, we show that the density-magnetic field cross-correlation $C\left(\delta n, \delta B_{\|}\right)$in the solar wind is $\simeq-1$ and increases slightly with ion plasma beta, $\beta_{i}$. Then we demonstrate excellent agreement with synthetic (eigenfunction) data in which less than $10 \%$ of the compressible energy is due to fast waves.

Measurements.- We use measurements from the Magnetic Field Investigation (MFI) 16] and the Three Dimensional Plasma (3DP) experiment [17] on the Wind spacecraft, in the unperturbed solar wind at $1 \mathrm{AU}$, during the years 1994-2004. The magnetic field is sampled at either 11 or 22 vectors/s (depending on the spacecraft telemetry rate) then averaged down to the spacecraft spin period $(3 \mathrm{~s})$. The ion moments are computed 


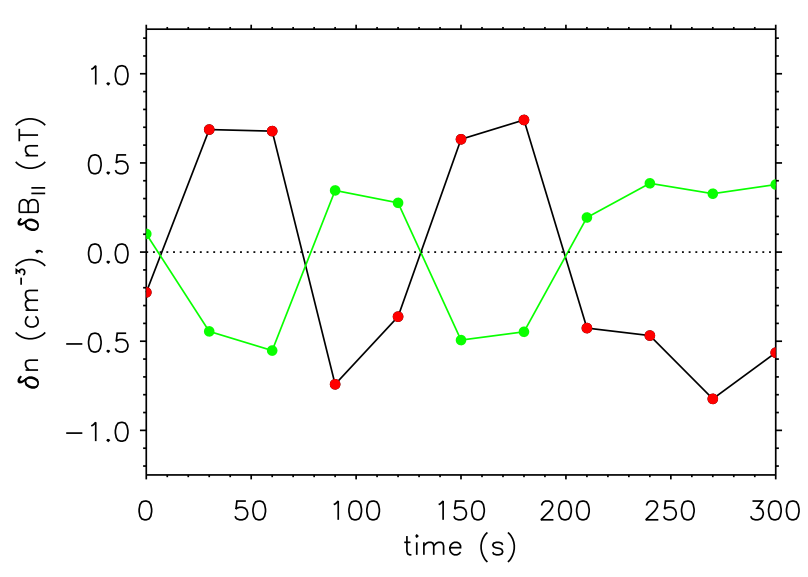

FIG. 1. Example of waveforms of proton density fluctuations $\delta n$ (black with red dots) and parallel magnetic field $\delta B_{\|}$(green) fluctuations. This interval has $\langle\delta n\rangle_{r} \sim 0.6 \mathrm{~cm}^{-3}$, $\left\langle\delta B_{\|}\right\rangle_{r} \sim 0.4 \mathrm{nT}$, and $C\left(\delta n, \delta B_{\|}\right) \sim-0.97$, however, even intervals with smaller density fluctuations exhibit significant anti-correlations.

on board the spacecraft at $3 \mathrm{~s}$ cadence; protons are separated from alpha particles by a fixed energy interval (which is occasionally adjusted in flight software) during the moment calculation; the solar wind alpha particle abundance is typically $3-5 \%$ of the proton number density. We select 1,089,491 300-s intervals of ambient solar wind data (corresponding to spatial intervals of approximately $L \approx 450 \mathrm{~km} / \mathrm{s} \times 300 \mathrm{~s}=135,000 \mathrm{~km} \sim 1350$ $\rho_{i}$, where $\rho_{i}$ is the ion Larmor radius) and the data is decimated by a factor of 10 (to $30 \mathrm{~s}$ cadence). Therefore, our data correspond to inertial range scales of approximately $k \rho_{i} \in\left(5 \times 10^{-3}, 5 \times 10^{-2}\right)$. After selection, the magnetic field data are averaged in 100-s windows to compute the local mean field $\mathbf{B}_{0}$. The fluctuation field $\delta \mathbf{B}$ is created by subtracting $\mathbf{B}_{0}$ and then rotated to a field-aligned coordinate (FAC) system defined by the $\mathbf{B}_{0}$ direction. In this new system, there is a compressible field fluctuation $\delta B_{\|}$and shear components $\delta B_{\perp, 1}$ and $\delta B_{\perp, 2}$. The most probable amplitude of the shear component $\delta B_{\perp}=\left(\delta B_{\perp, 1}^{2}+\delta B_{\perp, 2}^{2}\right)^{1 / 2}$ is approximately 3.4 times greater than most probable $\delta B_{\|}$and corresponds to the Alfvénic component of the turbulence.

Proton density data $\delta n=n-n_{0}$ is detrended over the same time intervals. Proton density is an integral over the $(3 \mathrm{~s})$ distribution function $f(\mathbf{v})$, computed from particle flux measurements in 16 individual energy channels [17. Since the counts are digitized discretely and the energy channels are also discrete (with $\Delta E / E \sim 0.2$ ), the $3 \mathrm{DP}$ proton density moments have a finite dynamic range. To assess this, we evaluated the joint probability distribution (not shown) of $\langle\delta n\rangle_{r}$ and $\left\langle\delta B_{\|}\right\rangle_{r}$, the RMS values. Below values of $\langle\delta n\rangle_{r} \approx 0.5 \mathrm{~cm}^{-3}$, the joint pdf reverts from being well-correlated to a broader set of values. Furthermore, the histogram of $\langle\delta n\rangle_{r}$ alone

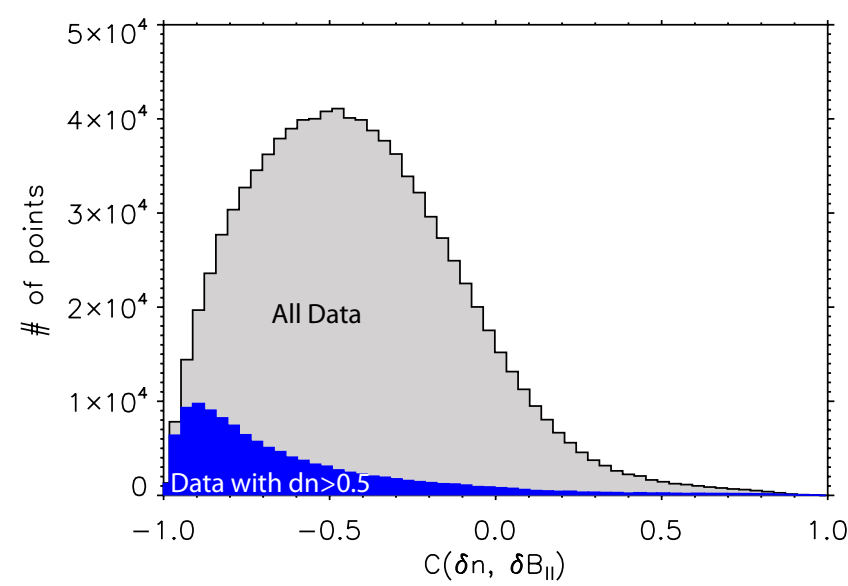

FIG. 2. Histogram of the cross-correlations $C\left(\delta n, \delta B_{\|}\right)$(grey, $1,089,491$ points total) and those above the measurement noise threshold $\delta n>0.5 \mathrm{~cm}^{-3}$ (blue, 119,512 points total).

shows clearly an artificial (non-Poisson) lower cutoff at around this value, a cutoff not seen in $\left\langle\delta B_{\|}\right\rangle_{r}$. We take $\langle\delta n\rangle_{r} \approx 0.5 \mathrm{~cm}^{-3}$ as the "noise" level of the density fluctuation measurement and restrict our analysis to intervals in which $\langle\delta n\rangle_{r} \geq 0.5 \mathrm{~cm}^{-3}$. This restriction reduces the dataset from 1,089,491 to 119,512 data intervals and biases our sample to higher absolute densities. The most probable values of absolute density are $\sim 4 \mathrm{~cm}^{-3}$ for the full and $\sim 12 \mathrm{~cm}^{-3}$ for the thresholded dataset. The distribution of plasma $\beta_{i}$ is unaffected by the thresholding.

We compute the normalized, zero-lag cross-correlation $C\left(\delta n, \delta B_{\|}\right)=\left\langle\delta n \delta B_{\|}\right\rangle /\langle\delta n\rangle_{r}\left\langle\delta B_{\|}\right\rangle_{r}$, which has a range from -1 to 1 . As described qualitatively above, we expect that $C\left(\delta n, \delta B_{\|}\right)$will be negative (positive) for slow(fast-) mode MHD fluctuations (see Figure 1). Figure 2 shows the distribution of $C\left(\delta n, \delta B_{\|}\right)$, both for all of the data and for the restricted dataset that exceeds the density noise threshold $\langle\delta n\rangle_{r} \geq 0.5 \mathrm{~cm}^{-3}$. While the entire dataset peaks below $0\left(\right.$ at $\left.C\left(\delta n, \delta B_{\|}\right) \simeq-0.5\right)$, the data with well resolved density amplitude levels peaks at $C\left(\delta n, \delta B_{\|}\right) \simeq-0.9$. Figure 3 shows the joint histogram of $C\left(\delta n, \delta B_{\|}\right)$vs. ion plasma beta $\beta_{i}$. The top panel shows the distribution of points, with a histogram of $\beta_{i}$ overplotted. The middle panel is the joint histogram normalized to number of $\beta_{i}$ points in each $\beta_{i}$ bin. This shows clearly that $C\left(\delta n, \delta B_{\|}\right)$is near -1 over the entire interval, increasing slightly with $\beta_{i}$ to $\simeq-0.7$ at $\beta_{i}=10$. In the bottom panel, the cumulative distribution shows that fewer than $10 \%$ of the intervals have $C\left(\delta n, \delta B_{\|}\right)>0$.

Synthetic Data.- A cubic synthetic plasma volume spanning scales $3 \times 10^{-3} \leq k \rho_{i} \leq 4.8 \times 10^{-2}$ is constructed using a $32^{3}$ grid. A spectrum of linear waves, with $90 \%$ of the energy in Alfven waves and the remaining $10 \%$ in a mixture of kinetic fast and slow waves, consistent with the observed $k^{-5 / 3}$ one-dimensional energy spectrum of the magnetic field fluctuations $|\delta \mathbf{B}|$, is created in the vol- 


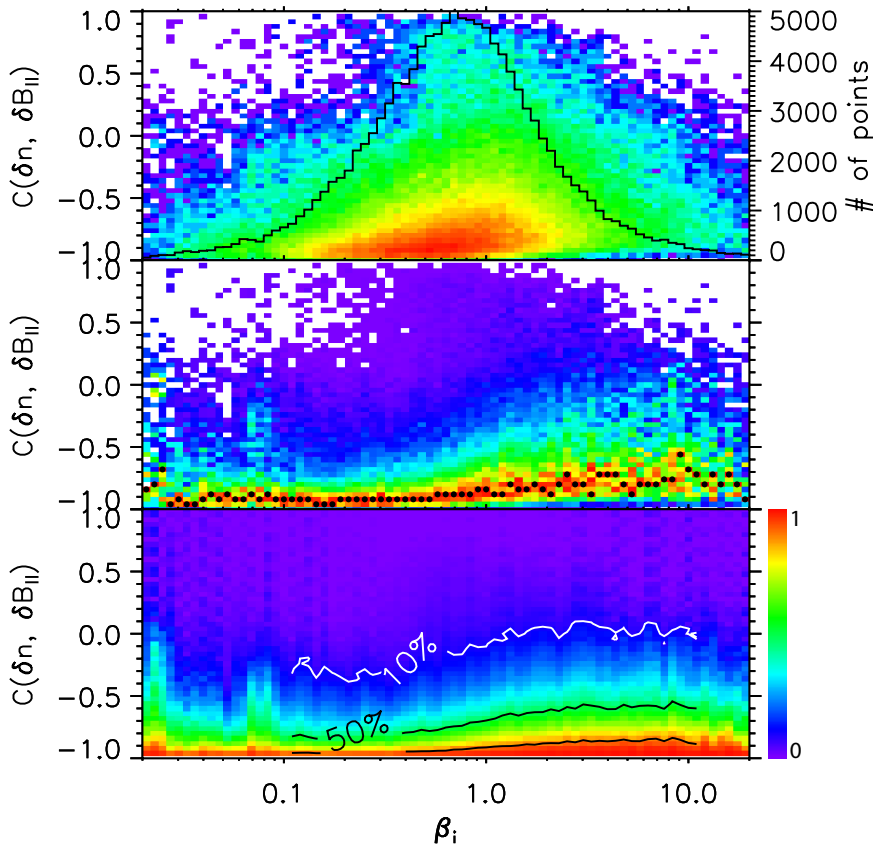

FIG. 3. (Upper) Total distribution of the $C\left(\delta n, \delta B_{\|}\right)$crosscorrelation as a function of ion plasma beta $\beta_{i}$. The count in each $\beta_{i}$ bin is overplotted (with the scale on the right). (Middle) Joint distribution of the $C\left(\delta n, \delta B_{\|}\right)$cross-correlation normalized within each $\beta_{i}$ bin. Black dots are the peak values in each $\beta_{i}$ bin. (Lower) Cumulative distribution of $C\left(\delta n, \delta B_{\|}\right)$ with contours at $90 \%, 50 \%$, and $10 \%$. These distributions consist of 119,512 independent data intervals.

ume using the linear eigenfunctions for these modes from the Vlasov-Maxwell linear dispersion relation [18, 19]; see Klein et al. 2011 [3] for more details. A fully-ionized proton and electron plasma is assumed, with isotropic Maxwellian velocity distributions, a realistic mass ratio $m_{i} / m_{e}=1836$, equal ion and electron temperatures $T_{i}=T_{e}$, and non-relativistic conditions $v_{t i} / c=10^{-4}$. Taking the MHD limit $k \rho_{i} \ll 1$, under these conditions the normalized linear Vlasov-Maxwell eigenfrequency depends on only two parameters, $\omega /\left(k v_{A}\right)=\bar{\omega}\left(\beta_{i}, \theta\right)$, the ion plasma beta $\beta_{i}$ and the angle $\theta$ between the wavevector and the mean magnetic field [3]. Once $\beta_{i}$ has been chosen, one needs only to specify the distribution of energy in wavevector space. Compressible MHD turbulence simulations generate an isotropic distribution of fast waves and critically balanced distributions of Alfvén and slow waves [20]. Therefore, we initialize the fast wave energy isotropically, while the Alfvén and slow wave energy mimics a critically balanced distribution by setting all modes with $k_{\|}>k_{0}^{1 / 3} k_{\perp}^{2 / 3}$ to zero, where $k_{0}$ corresponds to the scale of the plasma volume.

Time series of density and parallel magnetic field fluctuations are constructed by sampling the synthetic data at a probe moving through the volume at an oblique angle with respect to the mean field (tests have confirmed in-

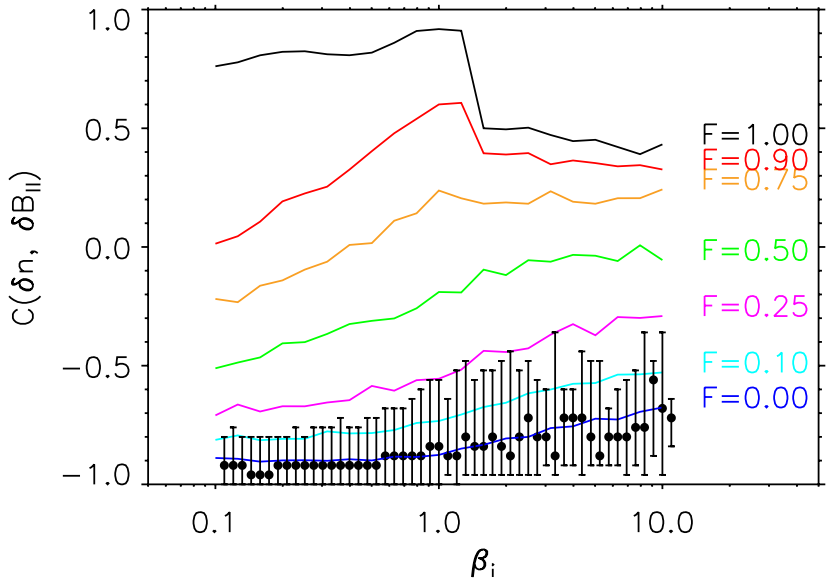

FIG. 4. Comparison of measured values of the $C\left(\delta n, \delta B_{\|}\right)$ cross-correlation (black dots with FWHM error bars) to the synthetic data predictions for the ratio of kinetic fast wave to total compressible energy $F$. Best agreement is with $F=0.00$, indicating that the compressible component of solar wind turbulence is almost entirely in the kinetic slow mode.

sensitivity to the choice of angle). We then compute the cross-correlation $C\left(\delta n, \delta B_{\|}\right)$as above. Figure 4 shows $C\left(\delta n, \delta B_{\|}\right)$for several values of the ratio of fast wave energy to total compressible energy $F$ vs. ion plasma $\beta_{i}$. Peak histogram values (and FWHM error bars) are also shown. The solar wind data are in striking agreement with the synthetic data $F=0.00$ curve.

Discussion.- Figure 4 shows that the observed correlation is consistent with a statistically negligible kinetic fast wave energy contribution for the large sample used in this study. Note, however, that a very small fraction of the data intervals have a positive cross-correlation (see Fig. 2), possibly indicating the presence of kinetic fast waves in these intervals.

Previous analyses have generally dismissed the possibility of kinetic slow waves because, in an isotropic Maxwellian plasma with warm ions, the collisionless damping via free-streaming along the magnetic field is strong [4]. However, in the limit $k_{\perp} \gg k_{\|}$applicable to a critically balanced power distribution, the damping rate of the slow waves is proportional to the parallel component of the wavevector, $\gamma \propto k_{\|}[19]$. For exactly perpendicular wavevectors, the damping rate drops to zerothis perpendicular limit of the slow wave corresponds to an undamped, non-propagating pressure-balanced structure (PBS). In compressible, strong MHD turbulence, it has been shown that the slow modes are cascaded passively by the Alfvénic turbulence [21, 22], so the energy cascade rate is related not to the slow wave frequency, but to the Alfvén wave frequency. Therefore, the more nearly perpendicular slow waves (possibly with $k_{\|}$well below critical balance, $k_{\|} \ll k_{0}^{1 / 3} k_{\perp}^{2 / 3}$ ) may be cascaded to smaller scales on the timescale of the Alfvénic tur- 


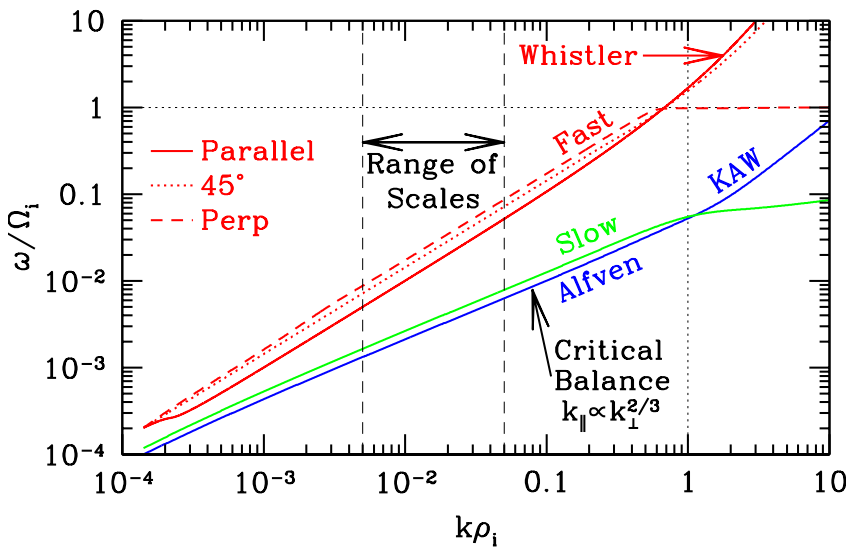

FIG. 5. Plot of frequency $\omega / \Omega_{i}$ vs. wavenumber $k \rho_{i}$ for the collisionless versions of the fast MHD (red), Alfvén (blue), and slow MHD (green) waves determined using the VlasovMaxwell linear dispersion relation. The range of scales considered in this study is indicated. Our measurements suggest that the solar wind spectrum lies on the blue-green curves.

bulence, while the collisionless damping of these modes remains weak.

We offer the following physical model of the compressible fluctuations in solar wind turbulence. At inertial range scales $k \rho_{i} \lesssim 0.1$, the density and parallel magnetic field fluctuations arise mainly from the kinetic counterparts of the fast and/or slow MHD waves. The measured $C\left(\delta n, \delta B_{\|}\right)$cross-correlation at these scales demonstrates that the compressible fluctuations are statistically dominated by kinetic slow mode fluctuations, and the distribution of power in wavevector space of these slow modes mimics the critically balanced distribution expected of Alfvénic fluctuations [3]. These kinetic slow wave fluctuations may be cascaded as passive fluctuations to smaller scales by the Alfvénic turbulence [21, 22], and so are predicted to exist down to the scale of the ion Larmor radius, and perhaps to even smaller scales. Thus, the evidence for PBSs over a range of timescales from $10^{3} \mathrm{~s}$ to $10 \mathrm{~s}$ [15] is explained by the presence of a distribution of kinetic slow waves that is undergoing a turbulent cascade to smaller scales driven by the Alfvénic turbulence. In addition, these kinetic slow modes suffer collisionless damping at a rate $\gamma \propto k_{\|}$, meaning that the more perpendicular the wavevector, the slower the damping rate.

The lack of a statistically significant fast wave component in the inertial range of solar wind turbulence has implications for the cascade of energy to small scales. Because the nonlinear energy transfer is believed to be dominated by local interactions in wavenumber space, significant nonlinear energy transfer occurs only between waves with similar linear frequencies. Fig. 5 shows the real linear frequencies $\omega$ of the collisionless counterparts of the MHD fast, Alfvén, and slow waves as a function of $k \rho_{i}$. For the isotropically distributed fast waves (red), we plot the parallel (solid), $45^{\circ}$ (dotted), and perpen- dicular (dashed) increase of the wavevector; slow (green) and Alfvén (blue) waves follow the critically balanced path $k_{\|}=k_{0}^{1 / 3} k_{\perp}^{2 / 3}$, with the isotropic driving scale $k_{0} \rho_{i}=10^{-4}$ for all cases. Since only the fast wave turbulent cascade is expected to nonlinearly transfer energy to whistler waves at $k \rho_{i} \gtrsim 1$, our analysis suggests that there is little or no transfer of large scale turbulent energy through the inertial range down to whistler waves at small scales.

Supported by NASA grants NNX10AC91G (Iowa) and NNX10AT09G (Berkeley) and by NSF grants AGS1054061 (Iowa) and AGS-0962726 (Berkeley).

[1] R. Bruno and V. Carbone, Living Reviews in Solar Physics 2, 4 (2005).

[2] W. Baumjohann and R. A. Treumann, Basic space plasma physics (London: Imperial College Press, 1996).

[3] K. G. Klein, G. G. Howes, J. M. TenBarge, S. D. Bale, C. H. K. Chen, and C. S. Salem, Astrophys. J.(2011), in preparation.

[4] A. Barnes, Phys. Fluids 9, 1483 (1966).

[5] C.-Y. Tu and E. Marsch, Space Science Reviews 73, 1 (1995).

[6] L. F. Burlaga and K. W. Ogilvie, Sol. Phys. 15, 61 (1970).

[7] M. Vellante and A. J. Lazarus, J. Geophys. Res. 92, 9893 (1987).

[8] D. A. Roberts, J. Geophys. Res. 95, 1087 (1990).

[9] W. H. Matthaeus, L. W. Klein, S. Ghosh, and M. R. Brown, J. Geophys. Res. 96, 5421 (1991).

[10] C.-Y. Tu and E. Marsch, J. Geophys. Res. 99, 21481 (1994).

[11] D. J. McComas, B. L. Barraclough, J. T. Gosling, C. M. Hammond, J. L. Phillips, M. Neugebauer, A. Balogh, and R. J. Forsyth, J. Geophys. Res. 100, 19893 (1995).

[12] D. B. Reisenfeld, D. J. McComas, and J. T. Steinberg, Geophys. Res. Lett. 26, 1805 (1999).

[13] B. Bavassano, E. Pietropaolo, and R. Bruno, Annales Geophysicae 22, 689 (2004).

[14] P. J. Kellogg and T. S. Horbury, Ann. Geophys. 23, 3765 (2005).

[15] S. Yao, J.-S. He, E. Marsch, C.-Y. Tu, A. Pedersen, H. Rème, and J. G. Trotignon, Astrophys. J. 728, 146 (2011).

[16] R. Lepping, Space Sci Rev 71, 207 (1995).

[17] R. P. Lin, Space Sci Rev 71, 125 (1995).

[18] E. Quataert, Astrophys. J. 500, 978 (1998).

[19] G. G. Howes, S. C. Cowley, W. Dorland, G. W. Hammett, E. Quataert, and A. A. Schekochihin, Astrophys. J. 651, 590 (2006).

[20] J. Cho and A. Lazarian, Mon. Not. Roy. Astron. Soc. 345, 325 (2003).

[21] J. Maron and P. Goldreich, Astrophys. J. 554, 1175 (2001).

[22] A. A. Schekochihin, S. C. Cowley, W. Dorland, G. W. Hammett, G. G. Howes, E. Quataert, and T. Tatsuno, Astrophys. J. Supp. 182, 310 (2009). 